\title{
Physikalische Therapie: Therapiemittel Wärme und Kälte (1)
}

\author{
Jean-Michel Jeannin
}

Basel, Schweiz

\section{Einleitung}

Die Anwendung der Therapiemittel Wärme und Kälte wird in der physikalischen Medizin als Thermotherapie bezeichnet. Umgangssprachlich wird jedoch unter Thermotherapie meistens nur die Wärmetherapie verstanden. De facto wird aber bei einer Thermotherapie Wärme oder Kälte mittels Medien angewendet, sodass von einer Wärme- und Kälteträgertherapie gesprochen werden soll [1]. Die Therapiemittel Wärme und Kälte zeichnen sich im Unterschied zum Wasser [2] weniger durch eine Vielfalt von Anwendungsmöglichkeiten als durch eine Vielfalt der Träger (Medien) aus. Die einzelnen Medien weisen unterschiedliche Wirkqualitäten auf und werden bisweilen auch unterschiedlich angewendet. Entsprechend gestalten sich die Durchführung von klinischen Studien und die Bewertung der Ergebnisse sehr anspruchsvoll. Wegen der Fülle des verfügbaren Stoffs beschränkt sich der vorliegende Artikel auf die Theorie und die praktische Anwendung. Die Besprechung von klinischen Studien ist einer weiteren Folge vorbehalten [1].

\section{Funktionsweise}

Es wird zwischen zwei grundsätzlich verschiedenen Wirkprinzipien unterschieden [1]:

- Wärme oder Kälte wirkt unmittelbar auf das behandelte Gebiet, z.B. durch Hemmung oder Förderung der Durchblutung oder der Stoffwechselaktivität. Gegenregulationen des Organismus werden im Zusammenhang einer solchen Therapie als nicht erwünschte Wirkungen betrachtet. Typische Anwendungen dieses Prinzips bestehen im Auflegen von Kälte- oder Wärmepackungen, die über längere Zeit (mehrere Minuten bis Stunden) lokal begrenzt eingesetzt werden.

- Wärme oder Kälte löst Gegenregulationen und adaptive Antworten aus, welche die eigentliche Wirkung darstellen. Solche Anwendungen dauern sehr kurz (wenige Minuten) und werden häufig am ganzen Körper

\begin{tabular}{|c|c|}
\hline $\begin{array}{l}\text { Italienischer } \\
\text { Fango }\end{array}$ & $\begin{array}{l}\text { Mischung von Thermalwasser und Mineralschlamm } \\
\text { vulkanischen Ursprungs, der zur Reifung Algen und } \\
\text { Mikroorganismen zugegeben werden (organischer } \\
\text { Fango, gereifter Fango), bisweilen auch Paraffin [5]. }\end{array}$ \\
\hline $\begin{array}{l}\text { Anorganischer } \\
\text { Fango }\end{array}$ & $\begin{array}{l}\text { Aufschwemmung von feinst gemahlenem vulkani- } \\
\text { schem Gestein in Brauchwasser, Mineralwasser oder } \\
\text { Thermalwasser [5]. }\end{array}$ \\
\hline Heilerde & $\begin{array}{l}\text { Pulver aus Lössablagerungen. Wird mit kaltem Wasser } \\
\text { verrührt angewendet [4]. }\end{array}$ \\
\hline Moorbad & $\begin{array}{l}\text { In Wasser aufgelöster Torf. Torfbäder gehören zur } \\
\text { Balneotherapie und werden hier nicht weiter bespro- } \\
\text { chen [6]. }\end{array}$ \\
\hline
\end{tabular}

appliziert. Zur Entfaltung der Wirkung muss diese Art von Anwendung wiederholt (seriell) verabreicht werden. Ein typisches Beispiel hierfür ist das Saunabad.

\section{Wärme oder Kälte?}

Ob ein Medium Wärme oder Kälte zuführt, hängt davon ab, ob seine Temperatur über oder unter der Indifferenztemperatur liegt. Die Indifferenztemperatur ist die Temperatur, die keine Gegenregulation auslöst [3]. Sie beträgt für gasförmige Medien ca. $28^{\circ} \mathrm{C}$ und für flüssige oder breiige Medien ca. $36^{\circ} \mathrm{C}[1]$.

\section{Wärmeträgertherapie: Anwendungstechnik, Wirkungen, Indikationen, Kontraindikationen}

Gebräuchliche Medien zur Wärmetherapie sind Peloide (Fango, Lehm, Heilerde, Torf) (Tab. 1), Paraffin (meistens Handbad, auch als Zugabe zur Fangozubereitung), Wasser (Überwärmungsbad, Wickel) und Luft oder Dampf (Dampfbad, Saunabad). Merkmale eines für die Therapie geeigneten Wärmeträgers sind eine hohe Wärmekapazität und die Gewährleistung eines guten und gleichmässigen Wärmeübergangs. Diese Anforderung erfüllen Peloide mit

\section{KARGER \\ Fax +497614520714} Information@Karger.com www.karger.com (c) 2015 S. Karger GmbH, Freiburg

1015-0684/15/0276-0344\$39.50/0 


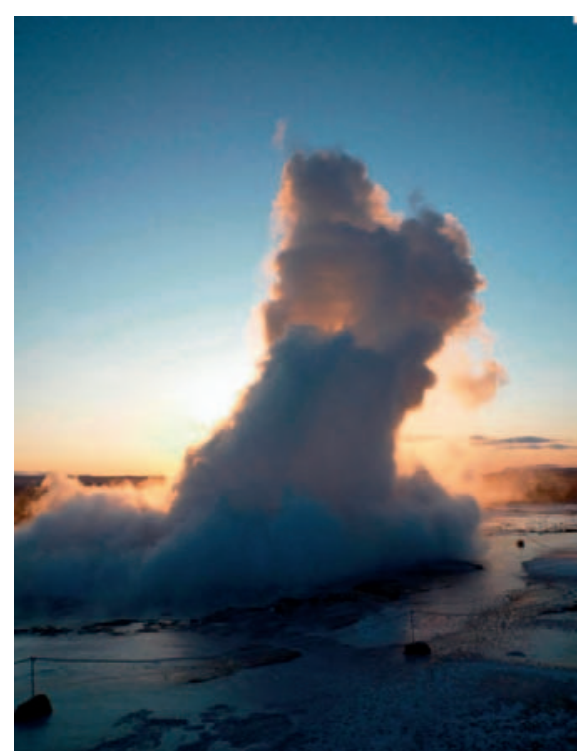

Abb. 1. Ein natürlicher Wärmeträger: Geysir auf Island, 2010 (Foto: Pascal Jeannin).

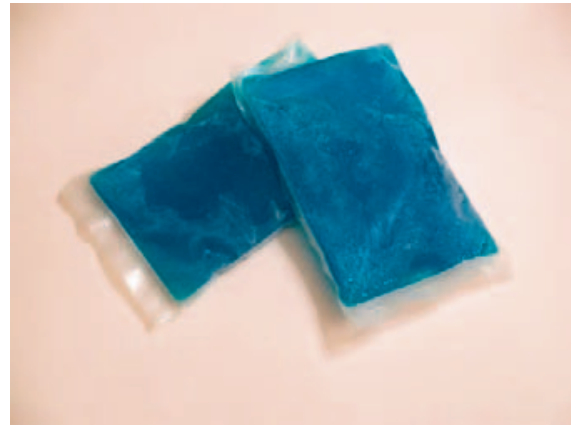

Abb. 2. Hot/Cold-Pack.

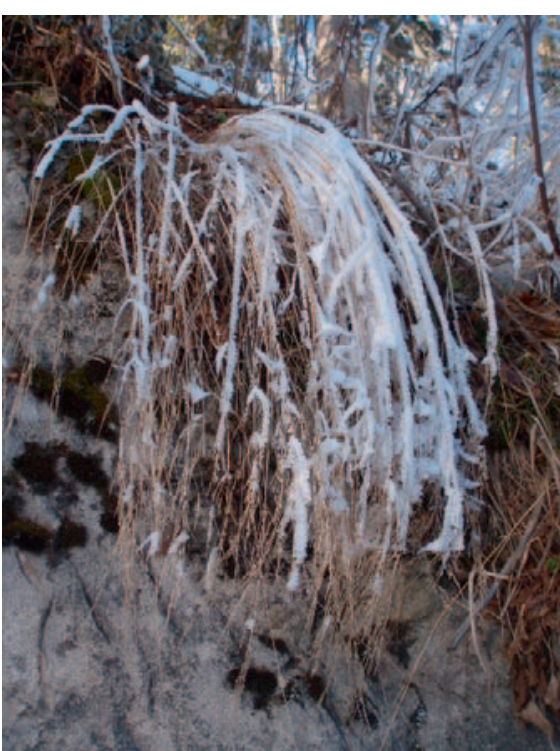

Abb. 3. Eis/Kälte. einer breiigen Konsistenz. Die Wärmekapazität (spezifische Wärme $\times$ Dichte) eines Peloids hängt von seinem Wassergehalt ab. Die grösste Kapazität haben Heilerde [4] und Badetorf (Wasseranteil etwa 30\%). Fango (pulverisiertes vulkanisches Gestein [5]) und Lehm haben eine etwas geringere Kapazität (ca. 40-45\% Wasser). Die geringste Wärmekapazität hat ein Moorbad [6] mit 95\% Wasseranteil [7]. Damit eine Packung bei heissem Kern an der Haut eine gut verträgliche Temperatur aufweist, muss sie mindestens $2 \mathrm{~cm}$ dick sein (flacher Temperaturgradient) [1]. Die lokale Therapie besteht in der Anwendung von Fango- (Tuff) oder Moorpackungen (wässrige Aufschwemmung von Torf). Die thermophysikalischen Eigenschaften ermöglichen die Anwendung von Temperaturen bis $50{ }^{\circ} \mathrm{C}$. Wasser lässt sich in verschiedener Weise als Wärmeträger verwenden (Abb. 1). Da die Grenzschicht zwischen Medium und Körperoberfläche sehr dünn ist, ist die maximal tolerierte Temperatur von $42{ }^{\circ} \mathrm{C}$ niedriger als bei einem Peloid [1]. Wasser wird auch mittels einer sogenannten heissen Rolle eingesetzt: Die Haut wird mit einem festgewickelten, mit heissem Wasser gefüllten Handtuch betupft oder abgerollt. Die Anwendungsdauer ist sehr kurz. Das Medium Luft gelangt in der Sauna zur Anwendung. Das Medium Dampf kann sowohl lokal als Dampfdusche als auch als Gesamtkörperbehandlung angewendet werden (Dampfsauna: Hamam, römisch-irisches Bad). Die thermodynamischen Eigenschaften des Mediums sind mehrheitlich vom Wasserdampfdruck abhängig. Mit steigender Feuchte nimmt der Wärmeübergang zu; entsprechend müssen die Anwendungstemperaturen gesenkt werden. Sie reichen von 30$36^{\circ} \mathrm{C}$ (Hamam, $100 \%$ relative Feuchte (RF) ) bis zu $85-95^{\circ} \mathrm{C}$ in der Sauna (5-10\% RF). Die Expositionsdauer nimmt mit steigender Temperatur ab (Hamam 3 h, Sauna 15 min) [1].
Eine lokale Wärmetherapie bewirkt eine Erhöhung der Hauttemperatur und der Hautdurchblutung, eine (lokale) Steigerung der Stoffwechselaktivität, eine reflektorische Muskelrelaxation sowie eine Linderung von namentlich muskulär bedingten Schmerzen. Mit einer Anwendung am Rumpf kann die glatte Muskulatur der inneren Organe (Magen, Darm, Gallenblase) auf reflektorischem Weg beeinflusst werden [1].

Eine systemische Wärmetherapie führt zu einer Erhöhung der Körperkerntemperatur, einer Beschleunigung des Pulses infolge Blutdruckabfalls durch Dilatation der Hautgefässe, einer Steigerung des Stoffwechsels sowie zu weiteren Reaktionen des vegetativen Systems. Wiederholte Anwendung eines Saunabads verbessert die unspezifische Abwehr [1].

Indikationen für eine systemische Wärmetherapie sind chronische generalisierte Schmerzen des Bewegungsapparats (Überwärmungsbad, Moorbad) sowie chronische Infektionsanfälligkeit und chronische Schmerzen (Sauna).

Kontraindikationen der Wärmeträgertherapie sind Herz-Kreislauf-Insuffizienz, Infektionen mit Fieber, Entzündungen und Verletzungen im zur Behandlung vorgesehenen Gebiet sowie unbehandelte Tumorkrankheiten und eine Hyperthyreose. Vorsicht ist geboten beim Orthostase-Syndrom: Es besteht die Gefahr eines Kreislaufkollapses, insbesondere bei hyperthermen Wannenbädern [1].

\section{Kältetherapie: Anwendungstechnik, Wirkungen, Indikationen, Kontraindikationen}

Als Kälteträger werden Luft, Wasser, Eis, Gase (gasförmiger oder flüssiger Stickstoff sowie Kohlendioxid) und 
Gele («Hot/Cold-Pack» (Abb. 2)) eingesetzt [1, 7]. Luft wird mittels einer Kältekammer angewendet (Cryosauna) [8]. Der unbekleidete Körper wird für 1-3 min Temperaturen von -70 bis $-120^{\circ} \mathrm{C}$ ausgesetzt. Die Akren (Hände, Füsse, Ohren) müssen hierbei vor der Kälte geschützt werden (Abb. 3) [1]. Wasser kann als Bad oder lokal als Kaltwasser- oder Eiswickel verabreicht werden [1]. Stickstoff und Kohlendioxid werden mittels spezieller Apparaturen meistens lokal angewendet [8]. Mit flüssigem Stickstoff wird eine Temperatur von $-196^{\circ} \mathrm{C}$ erzielt, mit flüssigem Kohlendioxid eine solche von $-75^{\circ} \mathrm{C}$ [8].

Mit einer lokalen Kryotherapie werden eine Drosselung der Durchblutung des behandelten Gebiets bis in tiefere Schichten und eine Analgesie bewirkt. Kälte wirkt ferner entzündungswidrig und gewebeabschwellend. Kurze starke Kältereize (Eisbäder, Eismassagen und Eisabreibungen) wirken spasmolytisch.

Indikationen für eine lokale Kälteträgertherapie sind akute lokale Schmerzen und Entzündungen allgemein sowie Krankheiten aus dem rheumatischen Formenkreis, im Besonderen ankylosierende Spondylitis, rheumatoide Arthritis, Osteoarthritis und Rückenschmerzen [8].

Indikationen für eine systemische Kältetherapie sind entzündliche und degenerative Erkrankungen des Bewegungsapparats, Osteoporose, spastische Lähmungen und multiple Sklerose [8].
Kontraindikationen für die Kälteträgertherapie sind: Kälteunverträglichkeit, Cryoglobulinämie, Cryofibrinogenämie, Agammaglobulinämie, Morbus Raynaud, offene Wunden, thromboembolische und entzündliche Venenkrankheiten, Hypothyreose, Malignome, akute Atemwegserkrankungen, gleichzeitige Behandlung mit Psychopharmaka sowie Alkoholmissbrauch.

\section{Schlussbemerkungen}

Die Therapiemittel Wärme und Kälte bieten eine breite Palette von Anwendungsformen zur Behandlung von Funktionsstörungen und Schmerzen des Bewegungsapparats und bestimmter innerer Organe. Sie reichen von der Sauna über das Kältebad bis zum Hot/Cold-Pack zur Selbstanwendung. Sie ermöglichen eine feinste Abstimmung (thermophysikalische Eigenschaften des Trägers, Temperatur, Dauer) auf die behandelte Person. Die Anwendungen sind wie bei allen Methoden der physikalischen Therapie sehr gut verträglich. Die Differenziertheit der Wärmeträger- und Kälteträger-Behandlung macht eine systematische Untersuchung in einer klinischen Studie aus methodischen Gründen, ähnlich wie in der Homöopathie, schwierig.

\section{Literatur}

1 Gutenbrunner C: 3.8 Wärme- und Kälteträger therapie; in Gutenbrunner C, Glaesener JJ: Rehabilitation, Physikalische Medizin und Heilverfahren. Heidelberg, Springer, 2007, p 75.

2 Jeannin J-M: Physikalische Therapie: Therapiemittel Wasser (1). Schweiz Z Ganzheitsmed 2015;27:92-95.
3 Glaesener JJ: 3.9 Hydrotherapie; in Gutenbrunner C, Glaesener JJ: Rehabilitation, Physikalische Medizin und Heilverfahren. Heidelberg, Springer, 2007, p 80.

4 Heilerde. Wikipedia. https://de.wikipedia.org/ wiki/Heilerde (aufgerufen am 25.10.2015).

5 Fango. Wikipedia. https://de.wikipedia.org/ wiki/Fango (aufgerufen am 25.10.2015).

6 Moorbad. Wikipedia. https://de.wikipedia.org/ wiki/Moorbad (aufgerufen am 25.10.2015).
7 Peloidtherapie. Wikipedia. https://de.wikipedia. org/wiki/Peloidtherapie (aufgerufen am 25.10. 2015).

8 Lubkowska A: Cryotherapy: physiological considerations and applications to physical therapy; in Bettany-Saltikov J (ed): Physical Therapy Perspectives in the 21st Century Challenges and Possibilities. Intech, 2012. 